\title{
Upper Bound of Primitive Exponent of a Class of Nonnegative Matrix Pairs*
}

\author{
Meijin LUO ${ }^{[1]} \quad \mathrm{Xi} \mathrm{LI}^{[2]}$ Sun $\mathrm{TAO}^{[3]}$ \\ ${ }^{[1]}$ School of Mathematics and Statistics, Hechi University Yizhou, Guangxi 546300, P.R. China \\ ${ }^{[2]}$ Department of Basic Education, Shanxi Yuncheng Vocational College of Agriculture, \\ Yuncheng,Shanxi 046012,P.R. China \\ ${ }^{[3]}$ Huaihai Industries Group Co.,Ltd,Shanxi 044000, P.R. China \\ ${ }^{[1]}$ meijin322@163.com, ${ }^{[2]}$ 924676854@qq.com, ${ }^{[3]}$ 279660136@qq.com
}

\begin{abstract}
Keywords: nonnegative; matrix pairs; exponent; upper bound
\end{abstract}
Abstract. There is a one-to-one relationship between nonnegative matrix pairs and two-colored digraph. With the knowledge of graph theory, by studying the associated directed digraph of a class of special nonnegative matrix pairs, that is a class of two-colored digraphs whose uncolored digraph have $n$ vertices and consists of one $n$-cycle and one $m$-cycle are considered. The upper bound of exponent and characteristic of extreme two-colored digraphs are given.

\section{Introduction}

Let $D$ be a digraph. A walk in $D$ of length $l$ is a sequence $v_{1}, v_{2}, \mathrm{~L}, v_{l+1}$ of vertices such that there is an arc in $D$ from $v_{i}$ to $v_{i+1}$ for $i=1,2, \mathrm{~L}, l$. The walk is a path if the vertices $v_{1}, v_{2}, \mathrm{~L}, v_{l+1}$ are distinct[1]. A two-colored digraph is a digraph whose arcs are colored red and blue. We allow loops and both a red arc and blue arc from $i$ to $j$ for all pairs $(i, j)$ of vertices. $D$ is strongly connected provided for each pair $(i, j)$ of vertices there is a walk in $D$ from $i$ to $j$. Given a walk $\omega$ in $D, r(\omega)$ (respectively, $b(\omega)$ ) is the number of red arcs (respectively, blue arcs) of $\omega$, and the composition of $\omega$ is the vector $(r(\omega), b(\omega))$ or $(r(\omega), b(\omega))^{T}$.

A two-colored directed digraph $D$ is primitive if and only if there exist nonnegative integers $h$ and $k$ with $h+k>0$ such that for each pair $(i, j)$ of vertices there is a $(h, k)$-walk in $D$ from $i$ to $j$. $\mathrm{A}(h, k)$-walk from $i$ to $j$ consisting of $h$ red arcs and $k$ blue arcs. The exponent of the primitive two-colored digraph $D$, denoted $\exp (D)$, is defined to be the smallest value of $h+k$ over all such $h$ and $k$.

Let $C=\left\{\gamma_{1}, \gamma_{2}, \mathrm{~L}, \gamma_{l}\right\}$ be the set of cycles of $D$. Set $M$ to be the $2 \times l$ matrix whose $i$ th column is the composition of $\gamma_{i}$. We call $M$ the cycle matrix of $D$. The content of $M$, denoted content $(M)$, is defined to be 0 if the rank of $M$ is less than 2 and the greatest common divisor of all $2 \times 2$ minors of $M$, otherwise.

Lemma 1.1 Let $D$ be a two-colored digraph having at least one red arc and one blue arc. Then $D$ is primitive if and only if $D$ is strongly connected and content $(M)=1^{[2]}$.

In fact, there is a one-to-one relationship between nonnegative matrix pairs and two-colored digraphs, so the problem of matrices can be changed into that of graphics to be solved. With the knowledge of graph theory, by studying the exponent of associated directed digraph of nonnegative matrix pairs, that is two-colored directed digraphs, the problem of nonnegative matrix,such as, the upper bound of primitive exponent, the lower bound of primitive exponent, the set of primitive exponent, the characterizations of extremeal digraphs etc can be solved.The concept of exponent of 
nonnegative matrix pair arises in the study of finite Markov chains [2-3], and some results have already been obtained[1-9].

In this paper, for $n>m \geq 2$, g.c.d $\{n, m\}=1($ g.c.d $\{n, m\}$ is the greatest common of $n$ and $m$ ), we consider the two-colored digraph $D$ in Fig.1 with $n$ vertices which has at least one red and one blue $\operatorname{arc}$

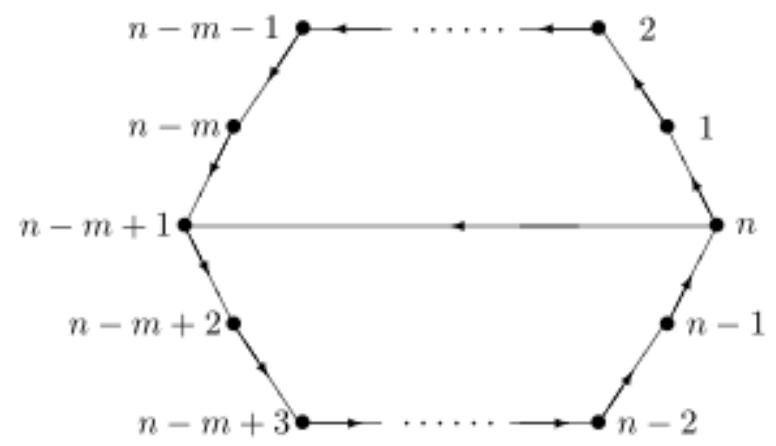

Fig.1 Uncolored digraph of $D$

Clearly, $D$ has only two cycles that one is an $n$-cycle and the other one is an $m$-cycle. Two cycles in $D$ only contains one non-common arc $n \rightarrow n-m+1$. Without loss of generality, we can assume that the cycle matrix of $D$ is

$$
M=\left[\begin{array}{cc}
a & b \\
n-a & m-b
\end{array}\right]
$$

for some integers $a$ and $b$.

Theorem 1.2 Let $D$ be primitive. Then if and only if $a m-b n= \pm 1$.

Proof From $(1), \operatorname{det}(M)=a m-b n$. By Lemma 1.1, D is primitive if and only if content $(M)$ $=1$, that is, $\operatorname{det}(M)= \pm 1$. Then the theorem follows.

By Theorem 1.2, we obtain the $n$-cycle of $D$ contains $a$ red arcs and $n-a$ blue arcs, and the $m$-cycle of $D$ contains exactly $b$ red arcs and $m-b$ blue arcs. If $a m-b n=1($ respectively $a m-b n=-1)$, then the inverse matrix of $M$ is

$$
M^{-1}=\left[\begin{array}{cc}
m-b & -b \\
-(n-a) & a
\end{array}\right] \text { or } M^{-1}=\left[\begin{array}{cc}
-(m-b) & b \\
n-a & -a
\end{array}\right] \text {. }
$$

\section{The bounds on the exponents}

Theorem 2.1 Let $a m-b n=1$ and $D$ be primitive, then

$$
\exp (D) \leq 2 a m n-a b n-a^{2} m \text {. }
$$

Proof We only prove that between each pair $(i, j)$ of vertices of $D$ there is an $\left(a^{2}(m-b)+a b(n-a), 2 a(m-b)(n-a)\right)$ - walk. For any pair $(i, j)$ of vertices of $D$, let $P_{i j}$ be the shortest path in $D$ from $i$ to $j$, and denote $r\left(P_{i j}\right)=s$ and $b\left(P_{i j}\right)=t$. We see that

$$
\begin{gathered}
{\left[\begin{array}{l}
s \\
t
\end{array}\right]+(a(m-b)-(m-b) s+b t)\left[\begin{array}{c}
a \\
n-a
\end{array}\right]+(a(n-a)+(n-a) s-a t)\left[\begin{array}{c}
b \\
m-b
\end{array}\right]} \\
=\left[\begin{array}{c}
a^{2}(m-b)+a b(n-a) \\
2 a(m-b)(n-a)
\end{array}\right] .(2)
\end{gathered}
$$

It is clear that $0 \leq s \leq a, 0 \leq t \leq n-a$. We consider the following four cases:

Case 1: $P_{i j}$ doesnot contain the vertex of $m$-cycle.

Because $1 \leq i \leq j \leq n-m$, then $0 \leq s \leq a-b, 0 \leq t \leq n-a-m+b$. Of course $a(m-b)-(m-b) s+b t$ $>0$ and $a(n-a)+(n-a) s-a t>0$.By (2), the walk that starts at vertex $i$, follows $P_{i j}$ to vertex $j$, 
goes $a(m-b)-(m-b) s+b t$ times around the $n-$ cycle, and $a(n-a)+(n-a) s-a t$ times around the $m$-cycle is an $\left(a^{2}(m-b)+a b(n-a), 2 a(m-b)(n-a)\right)$ - walk from $i$ to $j$.

Case 2: $P_{i j}$ contains the vertex of $m$-cycle.

Of course $0 \leq s \leq a, 0 \leq t \leq n-a, a(m-b)-(m-b) s+b t \geq 0$ and $a(n-a)+(n-a) s-a t \geq 0$. By (2), the walk that starts at vertex $i$, follows $P_{i j}$ to vertex $j$, goes $a(m-b)-(m-b) s+b t$ times around the $n$-cycle, and $a(n-a)+(n-a) s-a t$ times around the $m$-cycle is an $\left(a^{2}(m-b)+a b(n-a)\right.$, $2 a(m-b)(n-a))$ - walk from $i$ to $j$.

Hence there is an $\left(a^{2}(m-b)+a b(n-a), 2 a(m-b)(n-a)\right)$ - walk between each pair $(i, j)$ of vertices in $D$. So

$$
\exp (D) \leq a^{2}(m-b)+a b(n-a)+2 a(m-b)(n-a)=2 a m n-a b n-a^{2} m .
$$

The theorem follows.

As the proof of Theorem 2.1, we have the following Theorem 2.2.

Theorem 2.2 Let $a m-b n=-1$ and $D$ be primitive, then

$$
\exp (D) \leq b n^{2}-a b n+a m n-a^{2} m \text {. }
$$

\section{The extreme two-colored digraphs}

Theorem 3.1 Let $D a m-b n=1$ and $D$ be primitive, then $\exp (D)=2 a m n-a b n-a^{2} m$ if and only if the $a$ red arcs are consecutive on the $n$-cycle, that is, the $n-a$ blue arcs are consecutive on the $n$-cycle.

Proof Sufficiency: Form Theorem 2.1, we only proof $\exp (D) \geq 2 a m n-a b n-a^{2} m$.

Suppose that $(h, k)$ is a pair of nonnegative integers such that for all pairs $(i, j)$ of vertices there is an $(h, k)$-walk from $i$ to $j$. By considering $i=j=n$, we see that there exist nonnegative integers $u$ and $v$ with

$$
\left[\begin{array}{l}
h \\
k
\end{array}\right] M=\left[\begin{array}{l}
u \\
v
\end{array}\right]
$$

Taking $i$ and $j$ to be the initial vertex and the terminal vertex of $a$ consecutive red arcs on the $n$-cycle, and this path has composition $(a, 0)$.Hence

$$
M z=\left[\begin{array}{c}
h-a \\
k
\end{array}\right]
$$

has a nonnegative integer solution. Necessarily,

$$
z=M^{-1}\left[\begin{array}{c}
h-a \\
k
\end{array}\right]=\left[\begin{array}{l}
u \\
v
\end{array}\right]-M^{-1}\left[\begin{array}{l}
a \\
0
\end{array}\right]=\left[\begin{array}{l}
u \\
v
\end{array}\right]-\left[\begin{array}{c}
a(m-b) \\
-a(n-a)
\end{array}\right] \geq 0 .
$$

So $u \geq a(m-b)$.Next take $i$ and $j$ to be the terminal vertex and the initial vertex of $a$ consecutive red arcs on the $n$-cycle, and this path has composition $(0, n-a)$.Hence

$$
M z=\left[\begin{array}{c}
h \\
k-(n-a)
\end{array}\right]
$$

has a nonnegative integer solution. Necessarily,

$$
z=M^{-1}\left[\begin{array}{c}
h \\
k-(n-a)
\end{array}\right]=\left[\begin{array}{l}
u \\
v
\end{array}\right]-M^{-1}\left[\begin{array}{c}
0 \\
n-a
\end{array}\right]=\left[\begin{array}{l}
u \\
v
\end{array}\right]-\left[\begin{array}{c}
-b(n-a) \\
a(n-a)
\end{array}\right] \geq 0 .
$$

So $v \geq a(n-a)$. Thus

$$
h+k=\left[\begin{array}{ll}
1 & 1
\end{array}\right] M\left[\begin{array}{l}
u \\
v
\end{array}\right] \geq\left[\begin{array}{ll}
n & m
\end{array}\right]\left[\begin{array}{l}
a(m-b) \\
a(n-a)
\end{array}\right]=2 a m n-a b n-a^{2} m .
$$


Necessity: We only need to prove that the $a$ red arcs are not consecutive on the $n$-cycle; or the $n-a$ blue arcs are not consecutive on the $n$-cycle then $\exp (D)<2 a m n-a b n-a^{2} m$.

For any pair $(i, j)$ of vertices of $D$, let $P_{i j}$ be the shortest path in $D$ from $i$ to $j$, and denote $r\left(P_{i j}\right)=s$ and $b\left(P_{i j}\right)=t$. We consider the walk that starts at vertex $i$, follows $P_{i j}$ to vertex $j$, goes $\rho_{1}$ times around the $n$-cycle, and $\rho_{2}$ times around the $m$-cycle. Taking $\rho_{1}=a(m-b)-b-(m-b) s+b t$ and $\rho_{2}=(a-1)(n-a)+(n-a) s-a t$. We see that

$$
\left[\begin{array}{l}
s \\
t
\end{array}\right]+\rho_{1}\left[\begin{array}{c}
a \\
n-a
\end{array}\right]+\rho_{2}\left[\begin{array}{c}
b \\
m-b
\end{array}\right]=\left[\begin{array}{c}
a^{2}(m-b)-a b+b(a-1)(n-a) \\
a(m-b)(n-a)-b(n-a)+(m-b)(a-1)(n-a)
\end{array}\right] .
$$

Noting that $0 \leq s \leq a, 0 \leq t \leq n-a$, it is easy to see that $\rho_{1} \geq 0$ and $\rho_{2} \geq 0$. If $s=a$, then $t \geq 1$ and if $t=n-a$ then $s \geq 1$. This gives

$$
\begin{aligned}
\exp (D) & \leq a^{2}(m-b)-a b+b(a-1)(n-a)+a(m-b)(n-a)-b(n-a)+(m-b)(a-1)(n-a) \\
& =2 a m n-a b n-a^{2} m-b n-m(n-a) \\
& <2 a m n-a b n-a^{2} m .
\end{aligned}
$$

Combining Theorem2.1, the theorem follows.

As in the proof of Theorem 3.1, we can prove the following theorems 3.2.

Theorem 3.2 Let $D a m-b n=-1$ and $D$ be primitive, then $\exp (D)=b n^{2}-a b n+a m n-a^{2} m$ if and only if the $a$ red arcs are comsecitove on the $n$-cycle; or the $n-a$ blue arcs are comsecitove on the $n$-cycle.

\section{References}

[1] R.A. Brualdi, H.J. Ryser,Combinatorial Matrix Theory,Encyclopedia of Mathematics and its Applications, vol. 39, Cambridge University Press,Cambridge, 1991.

[2] B.L. Shader, S. Suwilo, Exponents of nonnegative matrix pairs, Linear Algebra Appl, vol. 363 (2003),p. 275-293.

[3] A.Berman, R. Plemmons, Nonnegative Matrices in the Mathematical Science, Classics in Applied Mathematics. Vol. 9, SIAM,Philadelphia,PA,1994.

[4] Yubin Gao, Yanling Shao, Exponents of two-colored double directed cycles, Journal of Heilongjiang University (Natural Science Edition), vol 4(2004),p.55-58.

[5] Yubin Gao, Yanling Shao, Exponents of two-colored digraphs with two cycles, Linear Algebra Appl, vol. 407(2005),p.263-270.

[6] Yanling Shao, Yubin Gao, Liang Sun, Exponents of a class of two-colored digraphs, Linear and Multilinear Algebra, vol.53(2005),p.175-188.

[7] Meijin Luo, Yubin Gao Exponents of a class of two-colored digraphs, Journal of North University of China (Natural Science Edition), vol 28(2007),p.377-382.(In Chinese)

[8] Meijin Luo, Yubin Gao Exponents of a class of three-colored digraphs with three cycles,Journal of Shandong University (Science Edition), vol.43(2008),p.65-72.(In Chinese)

[9] Meijin Luo, Yubin Gao, Primitive exponent of a class of two-colored digraphs, Journal of North University of China (Natural Science Edition),vol.29(2008),p.95-100.(In Chinese) 